\title{
¿Giro Digital? DEsafíos de LOS PRESUPUESTOS PARTICIPATIVOS ARGENTINOS FRENTE A LA PANDEMIA DE COVID- 19
}

\section{Rocío Annunziata ${ }^{1}$}

rocio.annunziata@gmail.com

Universidad Nacional de San Martín, Argentina

Consejo Nacional de Investigaciones Científicas y Técnicas, Argentina

\section{Emiliano Arena ${ }^{2}$}

emiliano.arena@gmail.com

Centro de Implementación de Políticas Públicas para la Equidad y el Crecimiento,

Argentina

Universidad Metropolitana para la Educación, Argentina

\section{Uriel Basualdo Franchino ${ }^{3}$}

urielbasualdo@gmail.com

Universidad Torcuato Di Tella, Argentina

\begin{abstract}
Este artículo se propone analizar los efectos de la pandemia de Covid-19 en la implementación de los Presupuestos Participativos en Argentina. Se observa que gran parte de las experiencias han tenido que suspenderse, otras han continuado de manera presencial respetando protocolos sanitarios, y algunas otras comenzaron un proceso de transición hacia plataformas digitales. Sin embargo, el giro digital se revela como problemático por los recursos con los que cuentan los municipios y porque en la visión de los equipos municipales la participación online resulta insuficiente y no puede reemplazar a la participación cara a cara. El artículo es producto de un relevamiento realizado a funcionarios/as municipales de 41 ciudades de 11 provincias en Argentina.
\end{abstract}

Palabras clave: Presupuesto Participativo/ Pandemia/ Giro digital/ Deliberación/ Participación

\footnotetext{
1 Doctora en Estudios Políticos de la École des Hautes Études en Sciences Sociales de Paris y Doctora en Ciencias Sociales de la Universidad de Buenos Aires.

2 Licenciado en Ciencia Política, Universidad de Buenos Aires.

3 Licenciado en Estudios Internacionales (Universidad Torcuato Di Tella).
} 


\section{Digital TURN? ChaLLENGES OF PARTICIPATORY BUDGETING IN ARGENTINA IN THE FACE OF THE COVID-19 PANDEMIC}

This paper aims to analyze the effects of the Covid-19 pandemic on the implementation of Participatory Budgeting in Argentina. We observe that a large part of the experiences has had to be suspended, others have continued inperson respecting health protocols, and some others began a transition process towards digital platforms. However, the digital turn is revealed as problematic due to the resources available to municipalities and because in the vision of municipal teams, online participation is insufficient and cannot replace faceto-face participation. The article is the product of a survey conducted with municipal officials from 41 cities of 11 provinces in Argentina.

Key words: Participatory Budgeting/ Pandemic/ Digital Turn/ Deliberation/ Participation 


\section{Introducción}

La llegada del COVID-19 y las medidas de aislamiento y distanciamiento físico que se implementaron para enfrentarlo constituyeron un desafio crítico para la gestión pública en general y para las instancias de participación ciudadana, como los Presupuestos Participativos (PP), en particular. Gran parte de los PP que estaban en funcionamiento a nivel local en Argentina tuvieron que suspender sus actividades, ya fuera por la necesidad de redirigir recursos a otras políticas y urgencias, ya fuera por la imposibilidad de organizar reuniones de ciudadanas y ciudadanos en el territorio. Pero también en algunos casos se desarrollaron estrategias adaptativas y creativas para sostener la participación, como la puesta en marcha de reuniones regidas por protocolos sanitarios en aquellas localidades en las que la pandemia no tuvo impactos tan fuertes; o el giro hacia la digitalización de los procesos participativos.

El giro digital en los PP, por otra parte, ya se estaba perfilando desde algunos años antes de la pandemia como una tendencia creciente; pero, aunque el escenario de 2020 significó una importante presión en esta dirección, a los municipios que implementaban la política no les ha resultado sencilla ni evidente la transición.

Este artículo se propone ofrecer una mirada panorámica sobre las experiencias de los municipios argentinos que implementaban el PP durante la pandemia de COVID-19 y en particular, sobre el modo en que entienden, a partir de ésta, un posible giro digital en los procesos de participación. Conocer cómo los gobiernos locales experimentaron y se adaptaron a este contexto crítico resulta fundamental para repensar la participación en la sociedad post-pandemia.

\section{Presupuestos Participativos online y pandemia}

La tendencia a la inclusión de tecnologías o instancias online en los PP ha sido abordada por la literatura especializada. En 2018, por ejemplo, Pineda Nebot (2018) señalaba la existencia de más de 200 experiencias de PP que utilizaban tecnologías en su diseño institucional. En efecto, lo más frecuente ha sido el desarrollo de experiencias "híbridas", o sea, de PP que incorporan alguna herramienta o momento digital, pero mantienen otras etapas del proceso en formatos "presenciales". Para Coleman y Cardoso Sampaio (2016) estas experiencias constituyen una "innovación dentro de la innovación" en la medida en que combinan el uso de TICs con procesos ya de por sí innovadores en materias de gestión pública.

En la mayoría de los casos, se denomina "PPs digitales" a metodologías que permiten a los ciudadanos votar online para seleccionar los proyectos (Wampler, McNulty y Touchton, 2018; Coleman y Cardoso Sampaio, 2016) aunque sin modificar el proceso previo de asambleas "cara a cara" en las que se discuten ideas y se elaboran proyectos factibles. La opción del voto online tiende a aumentar la cantidad de participantes pues reduce costos, pero reproduce la desigualdad cuando hay persistencia de la brecha digital (Spada, Mellon, Peixoto y Sjoberg, 2016), y 
esto ocurre tanto en los PP como en instancias similares del tipo de las consultas populares online (Welp, 2017).

En términos generales, entre las ventajas de la participación online se suele mencionar la flexibilidad y la disminución de los costos, especialmente porque permite superar barreras físicas como la distancia geográfica o el tiempo (Strandberg y Grönlun, 2018). En cambio, entre sus aspectos problemáticos se destaca que podría aumentar la distancia entre los pobres y la clase media en cuanto al acceso al poder político (Touchton,Wampler y Spada, 2019).Wampler, McNulty y Touchton (2018) observan que al reemplazar los encuentros territoriales o "cara a cara" por instancias virtuales, los PP se orientan crecientemente hacia las clases medias y zonas urbanas y menos hacia las clases pobres y zonas rurales.

Un elemento que podemos identificar en los estudios sobre "PP digitales" es que resulta poco frecuente que se desarrolle el ciclo completo de manera online, en la medida en que una de las mayores desventajas de los formatos digitales son las dificultades para producir verdaderos intercambios deliberativos (Graham, 2010; Coleman y Moss, 2012; Hartz-Karp y Sullivan, 2014; Manosevitch, 2014). Pareciera que persiste una idea, aún no suficientemente conceptualizada, sobre el carácter irremplazable del "cara a cara" (Annunziata, 2016). Algunos análisis muestran que los formatos que sostienen todo el proceso online podrían tender a una menor fragmentación en los proyectos, aunque tengan dificultades para lograr la participación de los sectores más vulnerables (Annunziata, 2020). En todo caso, los estudios suelen referirse a procesos de deliberación asincrónicos, con participación por escrito con la dificultad que eso implica para mantener los hilos de las conversaciones (Annunziata, 2016). La pandemia atrajo la atención sobre plataformas para intercambios sincrónicos que aún no han sido exploradas suficientemente ${ }^{4}$.

Por otra parte, la pandemia ha afectado a la participación en sus distintas formas. Se han suspendido o aplazado 66 procesos electorales, así como plebiscitos o referéndums, entre los cuales sobresale la consulta popular constitucional de Chile que se iba a celebrar en abril de 2020 como consecuencia del estallido social previo (Hernández Trejo, 2020). Las instancias participativas y deliberativas que suponen la reunión de personas en asambleas o mesas de trabajo también se vieron muy afectadas, y en particular aquellas que conforman un ciclo con varias etapas de reuniones como los PP. Si bien la opción del giro digital, esto es, de transformar el proceso participativo en un proceso online o "híbrido" apareció como alternativa, esto resultó muy difícil de resolver en la crisis. Por ejemplo, un estudio sobre el impacto de la pandemia en Nepal muestra que ninguna ciudad fue capaz de implementar plataformas online para mantener el proceso participativo (Bhusal, 2020).

$4 \quad$ Un estudio experimental analiza el impacto del tiempo en la deliberación comparando formatos sincrónicos y asincrónicos y concluye que los últimos conducen a un mayor calidad deliberativa (Strandberg y Grönlun, 2018). 
La mayoría de los PP del mundo simplemente interrumpieron sus actividades, como ocurrió, por ejemplo, en Ciudad de México donde se decidió trasladar a 2021 los recursos asignados a la herramienta para 2020 (Hernández Trejo, 2020). No obstante, también tuvieron lugar algunas experiencias innovadoras, ciudades que se adaptaron bien al nuevo contexto o en las que incluso la pandemia misma fue el pretexto para fortalecer la participación. Así, por ejemplo, en la ciudad de Grenoble en Francia, la pandemia funcionó como un motor para lanzar una primera edición de este dispositivo orientado a pensar las formas de solidaridad del "mundo del después" (Cho, Jerôme y Maurice, 2021).

Basándonos en los trabajos mencionados identificamos una serie de variables para analizar los hallazgos del relevamiento sobre las experiencias y percepciones del giro digital: desigualdades de acceso (distintas dimensiones de la brecha digital), calidad de deliberación, carácter irremplazable del contacto cara a cara, facilidad/ flexibilidad de la participación, inclusión de grupos sociales, capacidad de generar resiliencia en un contexto crítico. Las mismas nos han servido, como se verá más adelante, para crear categorías a partir de las preguntas abiertas del cuestionario suministrado.

\section{Metodología}

Este trabajo se apoya en un relevamiento realizado en 41 municipios de 11 provincias argentinas (ver Figura 1). En la investigación apuntamos a relevar a todo el universo de casos de municipios que estaban implementando un PP antes de la pandemia o que tenían previsto comenzarlo en 2020, lo lograran o no. Nuestra unidad de análisis fue el municipio, de modo que solo buscamos tener una respuesta del cuestionario por equipo municipal.

Es importante señalar que actualmente se desconoce la cantidad exacta de municipios con PP que existen en Argentina, ya que se discontinuaron las instancias institucionales de articulación entre gobiernos locales que implementan esta política (Red Argentina de Presupuestos Participativos). Por ello, se construyó un universo de gobiernos locales a partir de distintos registros. Primero, se tomaron los registros utilizados en las dos olas de encuestas nacionales de PP (Arena y Martínez, 2013; UNGS, 2015) y el Atlas Mundial de Presupuestos Participativos (Dias, Enríquez y Júlio, 2019). Luego, se tomaron los registros de los encuentros nacionales de PP Joven realizados en 2016 (C.A.B.A.) y 2017 (Resistencia) organizados por la Secretaria de Asuntos Municipales de la Nación y Unicef Argentina, y los encuentros nacionales de PP organizados en 2019 (Gualeguaychú) y 2020 (virtual) por la Universidad Nacional de General Sarmiento, la Fundación Friedrich Ebert y la Asociación Argentina de Democracia Participativa. Finalmente, se testearon estos datos con los suministrados por la Dirección Nacional de Gobierno Abierto. A partir de la unificación de estos registros se contactó a los municipios para conocer el estado de la implementación del PP y decidir si correspondía o no incluirlos en este estudio. 
Los municipios relevados fueron: Baradero (Buenos Aires), Escobar (Buenos Aires), General Madariaga (Buenos Aires), La Matanza (Buenos Aires), La Plata (Buenos Aires), Lanús (Buenos Aires), Mercedes (Buenos Aires), Morón (Buenos Aires), Saavedra (Buenos Aires), San Miguel (Buenos Aires), Tandil (Buenos Aires), Trenque Lauquen (Buenos Aires), Tres Arroyos (Buenos Aires), Tres de Febrero (Buenos Aires), Vicente López (Buenos Aires), Zárate (Buenos Aires), Ciudad Autónoma de Buenos Aires, Colonia Caroya (Córdoba), Córdoba (Córdoba), Río Cuarto (Córdoba),Villa Carlos Paz (Córdoba), Bella Vista (Corrientes), Gobernador Virasoro (Corrientes), Santa Lucía (Corrientes), Concepción del Uruguay (Entre Ríos), Concordia (Entre Ríos), Gualeguaychú (Entre Ríos), Paraná (Entre Ríos), Victoria (Entre Ríos), San Salvador de Jujuy (Jujuy), General Pico (La Pampa), La Rioja (La Rioja), Godoy Cruz (Mendoza), Lavalle (Mendoza), Maipú (Mendoza), Mendoza (Mendoza), Posadas (Misiones), Rafaela (Santa Fe), Reconquista (Santa Fe), Rosario (Santa Fe) y Santa Fe (Santa Fe) $)^{5}$.

\section{Figura 1}

Municipios relevados

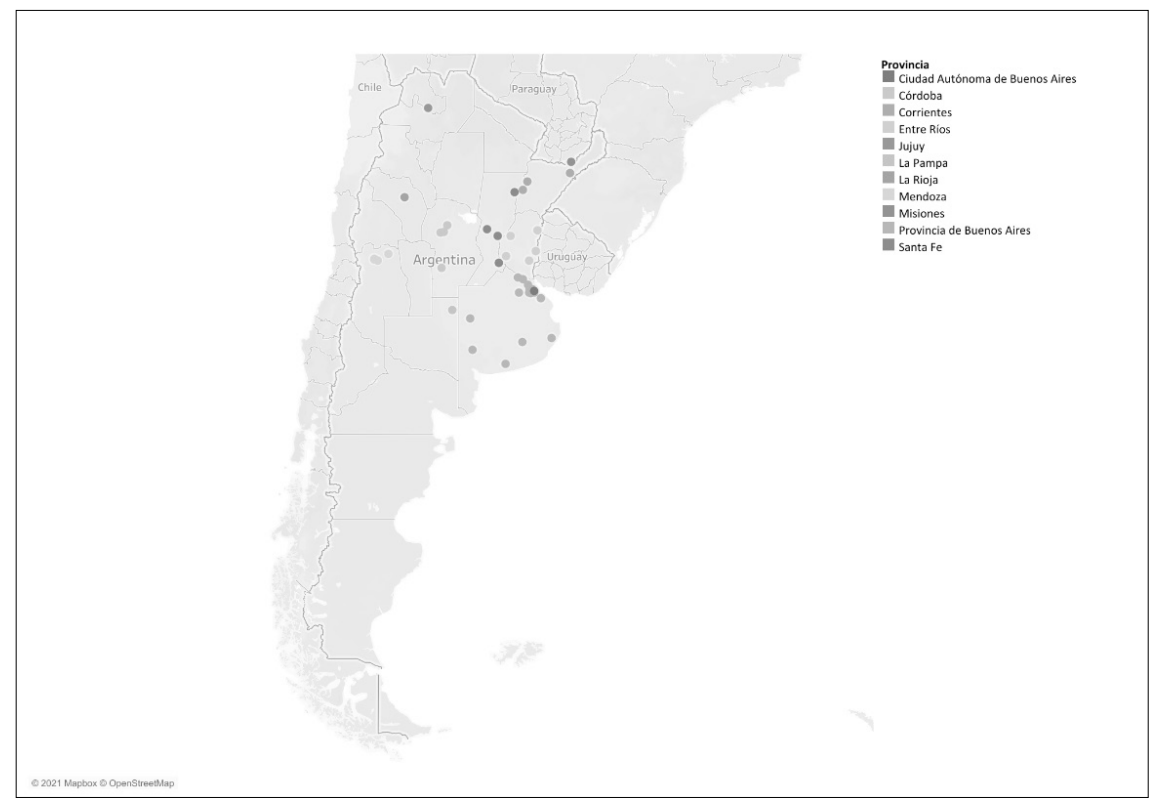

Fuente: elaboración propia sobre la base del relevamiento a municipios.

5 En el caso de la región patagónica los registros tomados como base señalaban la existencia de dos experiencias de PP: los municipios de Neuquén (Neuquén) y Viedma (Río Negro), que no respondieron en el plazo de cinco meses y fueron entonces descartados del estudio. 
Cabe mencionar que entre los municipios que respondieron al relevamiento contamos con 32 ciudades no capitales, 8 capitales provinciales y 1 capital de la nación (Ciudad de Buenos Aires). El tamaño de los municipios relevados fue muy variado: el más pequeño tiene 14.056 habitantes (Santa Lucía, Corrientes), mientras que la Ciudad de Buenos Aires, el caso de mayor tamaño, cuenta con 2.890.151 habitantes. La mayoría de las ciudades estudiadas tienen entre 100.000 y 150.000 habitantes (siendo la mediana de la muestra 123.871 habitantes).

La encuesta se diseñó con un cuestionario online y fue enviada a través de correos electrónicos a las/os funcionarias/os municipales identificadas/os previamente. El cuestionario contó, en su mayoría, con preguntas cerradas y, en menor medida, con preguntas abiertas. Una vez finalizado el proceso de relevamiento, se revisó la consistencia de la base de datos y se codificó con distintas categorías las respuestas a las preguntas abiertas, para dar cuenta de las variables teóricas mencionadas arriba.

El cuestionario fue respondido por un 55\% de mujeres y un $45 \%$ de varones, por funcionarias/os o representantes con distintos cargos en los equipos municipales, pero siendo siempre quienes estaban a cargo, en cada caso, de las políticas o mecanismos de participación de su municipio: 2 intendentes, 1 concejal, 4 secretarios/as, 1 subsecretario/a, 17 directores/as, 2 jefes/as de departamento, 5 coordinadores/as, 5 asistentes, además de 4 miembros de los equipos que prefirieron no difundir su cargo.

De manera complementaria, se realizaron entrevistas con expertos internacionales para contextualizar la situación de los PP argentinos en la pandemia con lo que se estaba observando en otras partes del mundo: Yves Cabannes, Nelson Dias y Giovanni Allegretti.

\section{Los Presupuestos Participativos argentinos frente a la pandemia}

\subsection{Efectos de la pandemia}

Lo primero que sobresale al analizar los resultados del relevamiento es que la pandemia de COVID-19 afectó mucho a los PP, redundando en la suspensión de casi la mitad de las experiencias. Esto puede representar un desafio a futuro ya que no es habitual que un municipio implemente esta política, la discontinúe y luego vuelva a implementarla ${ }^{6}$.

En efecto, como vemos en el Gráfico 1, un 41\% de los casos simplemente suspendió la implementación del PP, mientras que un $22 \%$ comenzó a retomarla, pero

\footnotetext{
Tal como Nelson Dias manifestó en la entrevista, en el mundo hubo una paralización significativa de los procesos de PP, y el mayor desafio en el contexto actual es retomarlos; en muchos casos simplemente no se logrará, pero donde sea posible es bueno mantenerlos por medios digitales para que no se interrumpan y los participantes sigan conectados (entrevista a Nelson Dias, 15-05-2020).
} 
implementando protocolos sanitarios y otro $22 \%$ optó por sostener las actividades de manera online. El 15\% restante se dedicó durante el año 2020 a ejecutar obras pendientes (como fue el caso deVicente López o de Paraná), adecuar la metodología para futuras implementaciones (como en la ciudad de La Rioja) o desarrolló estrategias especiales como la entrega domiciliaria de planillas para elaboración de propuestas (como sucedió, por ejemplo, en Saavedra).

\section{Gráfico 1}

¿Qué medidas se tomaron a partir de la pandemia?

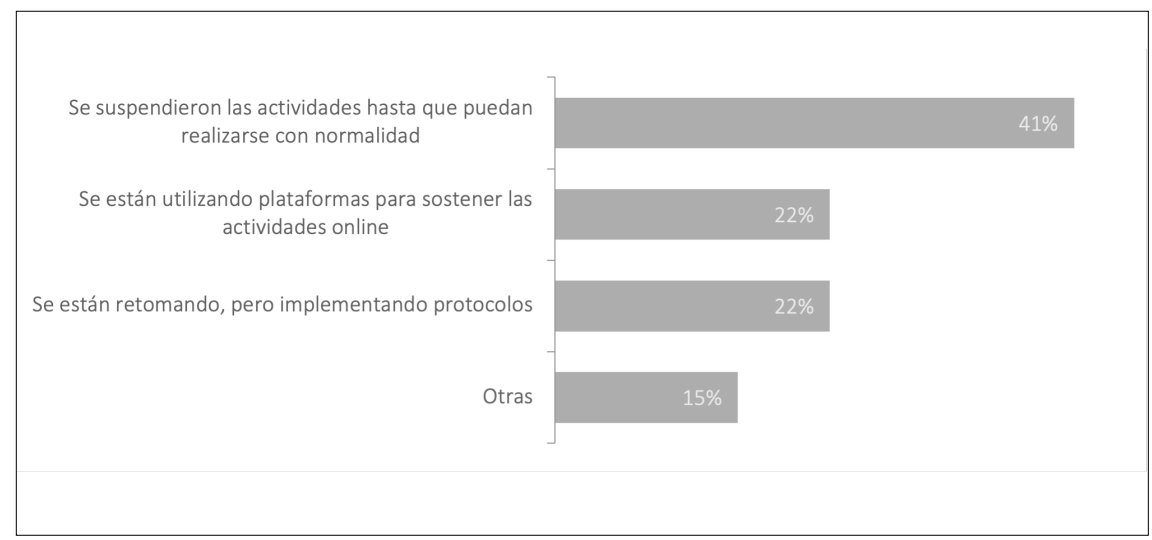

Fuente: elaboración propia sobre la base del relevamiento a municipios.

Otro impacto significativo de la pandemia sobre las experiencias de PP consistió en el impedimento de realizar encuentros "cara a cara", algo fundamental en estas instancias participativas que implican intercambios entre vecinos y con los funcionarios en reuniones o asambleas presenciales. Aunque la pandemia tuvo asimismo efectos negativos en los PP por la necesidad de los gobiernos locales de redirigir los recursos a la emergencia. Estas fueron las razones que llevaron a la suspensión en la mayoría de las experiencias, operando ambas en muchos casos (Gráfico 2). 
Gráfico 2

Razones para suspender la ejecución del PP

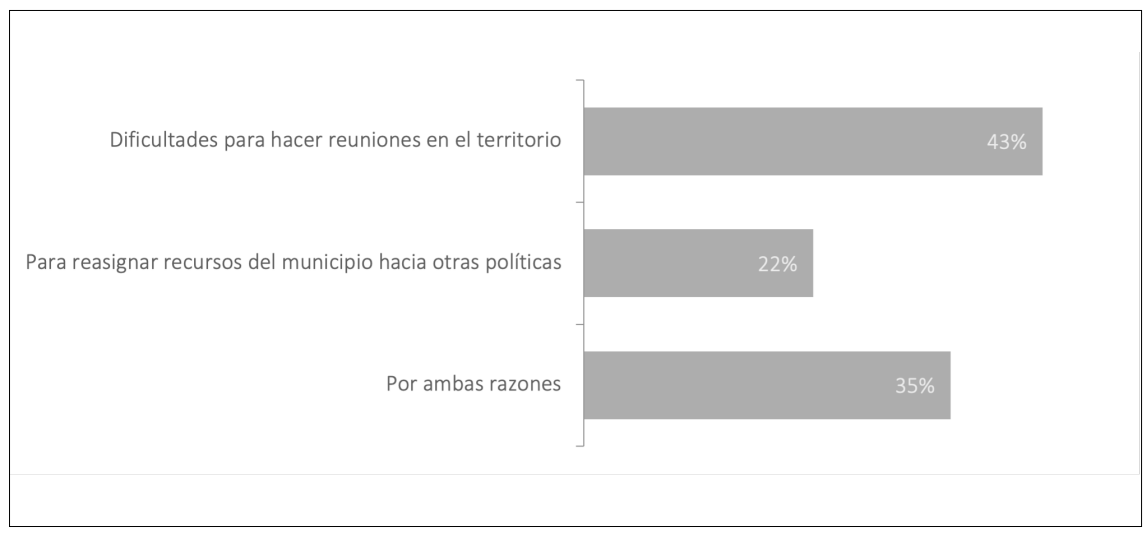

Fuente: elaboración propia sobre la base del relevamiento a municipios.

Los procesos de digitalización aparecieron en la pandemia como la solución evidente para muchas actividades de la vida cotidiana que no se podían realizar de manera presencial. Pero en el caso de los PP estos procesos no fueron mayoritarios, ya que la transición hacia formatos digitales se reveló complicada para los municipios. Muchos de los que usaron plataformas online ya las venían usando y otros incorporaron herramientas que se difundieron con la propia pandemia, como las plataformas para encuentros sincrónicos del tipo de Zoom, Google Meet o Jitsi. Este es un dato significativo porque se trata de plataformas que pueden en términos generales emplearse de modo gratuito, mientras que una de las dificultades para el giro hacia los formatos digitales en los municipios es el costo del desarrollo de plataformas propias o la adaptación de algunas existentes con código abierto, que requieren de todos modos de la expertise de programadores. Otros municipios utilizaron cuestionarios de Google, páginas web y redes sociales como Facebook o Whatsapp. La experiencia de General Pico, por ejemplo, combinó el uso de formularios digitales para crear propuestas con encuentros por Zoom.

Solo la ínfima minoría comenzó a implementar o diseñar software específicos para la participación ciudadana: en una de las experiencias comenzó a implementarse el PP a través de CIVICITI (Lanús) y en otra se inició el desarrollo de una plataforma propia (Victoria).

Los PP online en el mundo son mayormente experiencias híbridas, que combinan instancias o etapas presenciales con otras digitales. Lo cierto es que la etapa que más se ha experimentado de modo virtual es la votación, selección o priorización de proyectos. No obstante, las experiencias relevadas nos muestran que durante la pandemia el grupo de municipios que se volcó a la digitalización de sus procesos 
empleó las plataformas para: hacer propuestas y discutir proyectos (43\%), hacer propuestas y seleccionar proyectos (29\%), hacer propuestas (14\%), hacer propuestas, discutir proyectos y seleccionar proyectos (14\%).

\section{Dificultades en los procesos de giro digital}

Si bien el proceso de giro digital ya había comenzado antes de la pandemia y contribuyó a mantener la actividad participativa en un contexto de crisis, la gran mayoría de los equipos municipales que implementan el PP en Argentina consideraron que las plataformas digitales no son suficientes para reemplazar las instancias presenciales en los procesos de participación (Gráfico 3).

\section{Gráfico 3}

¿Cree que las plataformas online son suficientes para reemplazar las instancias presenciales?

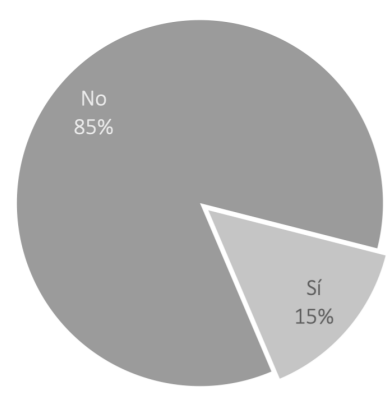

Fuente: elaboración propia sobre la base del relevamiento a municipios.

Las principales razones que mencionaron quienes consideran que las plataformas digitales no pueden reemplazar a las instancias presenciales son: las desigualdades de acceso (incluyendo en esto tanto las materiales -falta de dispositivos, débil conectividad, etc.- como las simbólicas o de capacidad de uso); la necesidad de contacto que supone la participación y que no puede suplir el formato online (entendiendo aquí que el contacto aporta a la participación tanto una dimensión afectiva como una dimensión informativa); y, en menor medida, la calidad de la deliberación (algo que siempre ha sido un desafio para las plataformas digitales) ${ }^{7}$. Algunos también mencionaron la complementariedad, es decir, consideraron que 
los procesos puramente online no son suficientes para una buena participación, sino que deben complementarse con instancias cara a cara. En el Gráfico 4 se observa la distribución de las razones por las que las plataformas digitales no pueden reemplazar el funcionamiento "cara a cara" del PP.

Gráfico 4

Razones por las cuales las plataformas digitales no son suficientes para el funcionamiento del PP

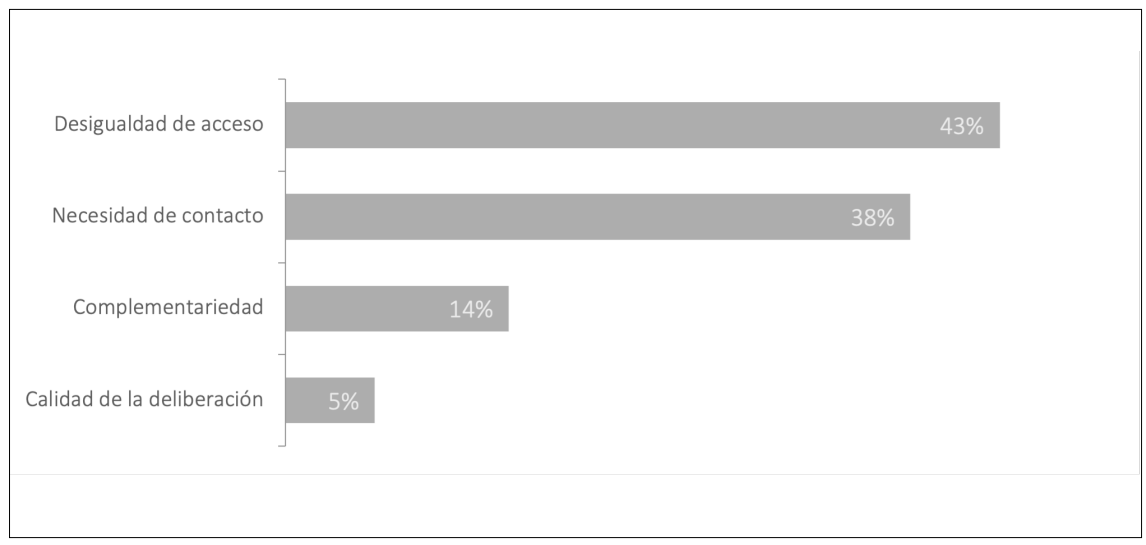

Fuente: elaboración propia sobre la base del relevamiento a municipios.

Con respecto a lo que hemos llamado "desigualdad de acceso", los equipos municipales subrayaron aspectos no solamente materiales. Por ejemplo, una funcionaria de Mercedes sostuvo que el formato online es insuficiente para la participación "...por la accesibilidad a la tecnología, tanto de las personas que viven en los barrios como de los adultos mayores". Los adultos mayores aparecieron claramente como un grupo con dificultades para adaptarse a una transición hacia la participación digital. Como afirmaban en el equipo de Saavedra: "Los de mayor edad quedan excluidos, en un alto porcentaje, en poder dar su opinión o propuesta". En el equipo municipal de Tandil se hizo referencia también a las desigualdades en términos de capacidades o conocimientos en el uso de las herramientas online: "debilita a la ciudadanía una instancia donde un ciudadano quizás no pueda participar por limitaciones tecnológicas".

Un funcionario del municipio de Córdoba afirmaba en el mismo sentido:“....adecuar la metodología presencial a la virtualidad no es nada fácil, sobre todo porque no todas las personas disponen de acceso (en términos de dispositivos, conectividad o de saber cómo participar) por lo cual se estaría restringiendo la participación, sobre

ciudadanas/os. Contrariamente a lo que sostuvo Nelson Dias, este especialista aboga por una desdigitalización de los procesos de PP (entrevista con Yves Cabannes, 12-05-2020). 
todo a los que no acceden a las TICs y que generalmente es población que desea y necesita respuestas más urgentes". De este modo, se llamó la atención sobre el hecho de que el formato online tiende a reforzar desigualdades preexistentes porque son los grupos más desfavorecidos, que podrían aprovechar las instancias de participación, quienes precisamente se ven excluidos por la falta de acceso en sentido amplio a las tecnologías.

Hay algunas zonas en las que las desigualdades de acceso no tienen solo una base social (socio-económica o etaria) sino territorial, como en las provincias de Mendoza o Misiones. Por ejemplo, en el caso de Lavalle se señalaba: "La conectividad no es buena en todo el territorio". También en el caso de Posadas: "...muchos barrios de la ciudad tienen problema de acceso a la tecnología". O en el caso de Maipú: "...tenemos en nuestro departamento habitantes de zonas rurales que no tienen acceso a la tecnología...".

Cabe observar que la pandemia misma ha recrudecido las desigualdades materiales de acceso que existían previamente, por ejemplo, en cuanto la cantidad de dispositivos disponibles en el grupo familiar. Como lo notaba un funcionario de Concordia: “...los celulares están siendo usados para las tareas escolares...".

La otra dimensión fundamental del carácter irremplazable de lo presencial es el contacto. Por ejemplo, el equipo del municipio de Río Cuarto sostenía: "El contacto es esencial para sostener un verdadero programa de participación ciudadana". En la misma línea, ampliaban del municipio de General Pico, que: "La presencialidad en territorio aporta información que de manera digital es imposible conseguir, no solo para este programa sino también para la gestión en su conjunto".

Como dijimos, el contacto presencial parece aportar una dimensión informativa, pero también una dimensión afectiva, fundamental para la participación que no puede reemplazar un formato online. El equipo de Gualeguaychú sostuvo que: “...nada reemplaza la llegada cara a cara a los barrios, los debates entre vecinos". El municipio de Morón aportaba lo siguiente: "En Morón armamos para 2020 un PP completamente descentralizado por barrios [...] Eso nos permitía darnos una lógica de proximidad, con el objetivo de generar mayor participación y relanzamiento de la política, que no es posible desde una plataforma online". En el mismo sentido afirmó una funcionaria del equipo de Trenque Lauquen:"La riqueza de la herramienta es el contacto directo con el vecino y la proximidad con los lugares que se desean intervenir con el dinero asignado". Una funcionaria del equipo de Vicente López afirmaba: "Considero un valor agregado muy importante el contacto con los ciudadanos y las organizaciones en las reuniones y talleres presenciales. El compromiso es mayor cuando se combinan ambas instancias.

Las plataformas online, como la que tenemos en Vicente López, ayudan sobre todo a la difusión pero no reemplazan el contacto cara a cara". En Reconquista el equipo observaba que el formato online no es en sí mismo suficiente "porque el contacto 
cara a cara con el vecino es fundamental para empatizar con el ciudadano, ponernos en su lugar a través de lo que él nos pueda relatar respecto de la necesidad que nos plantea. No es lo mismo una necesidad redactada en un renglón que el relato en primera persona de un problema con el que el vecino convive cotidianamente". Lo afectivo y lo informativo están conectados porque gracias a poder "ponerse en el lugar del ciudadano" se puede lograr una información con un carácter diferente. La dimensión afectiva del contacto es también el sentido de comunidad, como sostenían en el equipo de Godoy Cruz: "La interacción entre actores y municipio de manera presencial fortalece lazos humanos y ayudan a crear sentido de comunidad".

Es posible observar incluso una articulación entre lo que entendemos como la dimensión afectiva del contacto y la calidad de la deliberación. Tal como lo subrayaba una funcionaria del municipio de Zárate: "Los espacios presenciales generan confianza y la discusión grupal permite pasar de ideas individuales a construcciones colectivas".

Ahora bien, es importante destacar que, aunque la mayoría consideró que la participación online por sí sola resulta insuficiente, los funcionarios municipales entrevistados encontraron distintas ventajas y desventajas en el uso de plataformas digitales. Algunas de las ventajas han aparecido más claramente durante la pandemia (ver Gráfico 5).

\section{Gráfico 5}

Ventajas en el uso de plataformas online

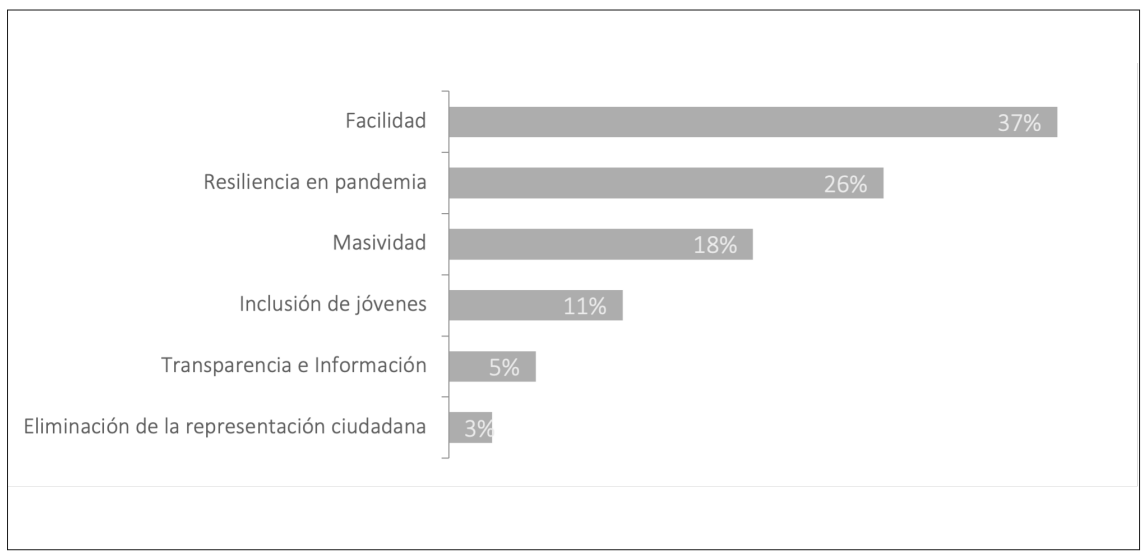

Fuente: elaboración propia sobre la base del relevamiento a municipios.

La principal ventaja mencionada fue la facilidad para participar que ofrecen las plataformas digitales. En esto se incluye tanto a la posibilidad de acortar distancias geográficas, como la simplificación de los procesos, la practicidad y la comodidad para participar, la reducción de recursos empleados, y la mayor frecuencia que 
permite este formato al no exigir el desplazamiento. Por ejemplo, el equipo municipal de Escobar sostenía: "La ventaja es que se pueden organizar encuentros sorteando distancias y con mayor frecuencia". Del mismo modo, en la ciudad de Córdoba se afirmaba: "Una ventaja puede ser acortar las distancias geográficas para participar en un taller, en cuanto a los ciudadanos / vecinos como para técnicos y funcionarios". La practicidad y comodidad fueron muy mencionadas por los equipos municipales, tomemos el caso de Paraná: "los vecinos y vecinas pueden votar desde cualquier lugar, y en cualquier momento, por ejemplo pueden hacerlo desde su horario laboral, las personas con movilidad reducida tendrían una opción para hacer valer su voluntad sin la necesidad de trasladarse. En conclusión: sus ventajas serían el tiempo y el espacio". El equipo de Gobernador Virasoro opinó por su parte: “...puede participar más gente por la masividad del uso de redes y mayor comodidad". El gobierno de Reconquista conectaba también la facilidad con el mayor número de participantes en los formatos digitales: "Puede ser más ágil y cómodo plantear una necesidad o problemática en una plataforma virtual que hacernos el tiempo de asistir a un taller de dos horas. Por esa misma rapidez y comodidad se puede llegar a más personas". Es interesante notar que, mientras que algunos formatos digitales pueden implicar mayores inversiones de recursos que los presenciales, otros, por el contrario, abaratan los costos para los municipios, como pudo ocurrir con las herramientas gratuitas que se usaron masivamente en la pandemia.

Luego, se menciona lo que denominamos "resiliencia en pandemia", es decir, el hecho de que estas plataformas hayan permitido resistir al contexto crítico del año 2020 logrando mantener los canales de participación abiertos aun sin ser los formatos ideales. En Concordia mencionaban, por ejemplo: "Se puede continuar con la comunicación aún en tiempos de pandemia". El equipo de Lavalle subrayaba como ventaja que "el contacto continúa" y el equipo de Mercedes también destacaba la "continuidad". En La Matanza sostuvieron, en la misma línea, que las herramientas digitales permitieron y permiten mantener las redes con los participantes habituales para que no se rompan del todo los lazos: "La ventaja es que se pueden seguir sosteniendo mínimamente vínculos sociales a través de las plataformas". Del mismo modo, en Rafaela afirmaron: "una ventaja es que cuando el vínculo es preexistente ayuda en este contexto". La "resiliencia en pandemia" es entonces una ventaja contextual, situacional.

Los equipos municipales de distintos gobiernos locales que no tenían ni la experiencia ni una expectativa particular en los formatos online de PP, vieron en éstos, sin embargo, un modo -si bien no ideal- de sostener el contacto y la comunicación, para evitar un cierre completo de los espacios participativos. La participación por medio de plataformas digitales también apareció en el contexto de la pandemia como una tendencia propia de la época, tal como se observaba en La Rioja: "Post pandemia, creo que los PP en modo online serán la nueva normalidad a la hora de elaborar agendas de trabajo colaborativo entre los diferentes actores de la sociedad (...) El trato personal es invalorable, siempre será la forma más asertiva para construir 
un vínculo, pero dada la situación mundial tenemos que amoldarnos a esta nueva forma de vida."

En tercer lugar, apareció como ventaja la masividad de la participación. Las plataformas online pueden lograr atraer, dependiendo del contexto territorial y del acceso a las tecnologías, una mayor cantidad de participantes, en la medida en que se adaptan a la vida cotidiana y pueden demandar un menor esfuerzo que los encuentros cara a cara. Como vimos más arriba, a partir de los testimonios de los municipios de Gobernador Virasoro y de Reconquista, la masividad se encuentra muy vinculada a la facilidad o comodidad en el uso de las redes, y parecería superar el que se presenta como el mayor obstáculo de la participación online, es decir, la desigualdad de acceso.

En cuarto lugar, entre las ventajas surgió la posibilidad de incluir mejor a los jóvenes en las instancias participativas. Este aspecto ha sido subrayado por distintos estudios: la participación online amplía el público de menores franjas etarias y de sectores medios con respecto a la participación presencial, mientras que reduce la de adultos mayores y sectores populares. Pero en aquellos municipios en los que se está buscando un mayor involucramiento de las juventudes, las plataformas digitales se revelan como una alternativa exitosa. El municipio de Concepción del Uruguay notaba que el formato online: "Aumenta considerable la participación de los jóvenes ciudadanos". Otros ejemplos de municipios que subrayaron esta ventaja son Lanús, cuyo equipo consideraba que lo online "permite incorporar a los más jóvenes que, normalmente, suelen estar poco presentes en los espacios presenciales", mientras en Godoy Cruz: "facilita la participación de jóvenes y los involucra en procesos democráticos."

Finalmente, un porcentaje menor de equipos municipales mencionaron que la posibilidad de ofrecer estadísticas actualizadas de participación y de obras, y en términos generales, la mayor transparencia de la gestión, son ventajas de las plataformas online. Éste es el caso de la Ciudad Autónoma de Buenos Aires y de Villa Carlos Paz, que subrayaban como ventaja el hecho de poder mantener a los vecinos informados sobre el avance de los proyectos, la transparencia y la generación de bases de datos que puedan emplearse para ir mejorando la participación. Esto es interesante dado que para los gobiernos municipales suele ser dificil y costoso llevar estadísticas de participación mientras que los formatos online pueden automatizar estos procesos de recolección de datos.

Un elemento que sobresale, al que pocas veces se presta atención en los estudios sobre participación online, es lo que hemos denominado como "eliminación de la representación”. Esto significa que las plataformas digitales permiten llegar más directamente a las personas no organizadas, fomentando una participación individual y no mediada. Esta fue la opinión del equipo de Godoy Cruz: "Iguala a los individuos como tales y pone límite a propuestas corporativas: en el sistema de asambleas organizaciones no gubernamentales se veían beneficiadas por sobre ciudadanos y ciudadanas no organizados, imponiendo muchas veces sus propuestas 
sobre la de una vecina o vecino. La digitalización actúa como un igualador dado que permite la misma capacidad de difusión de las diferentes propuestas". Lo que es juzgado aquí como una ventaja, como veremos, también es considerado una desventaja en otras experiencias.

Cuando pasamos a observar las desventajas señaladas por los equipos municipales (Gráfico 6), la principal resulta coincidente con la principal razón de la insuficiencia de la participación online que señalamos arriba.

\section{Gráfico 6}

Desventajas en el uso de plataformas online

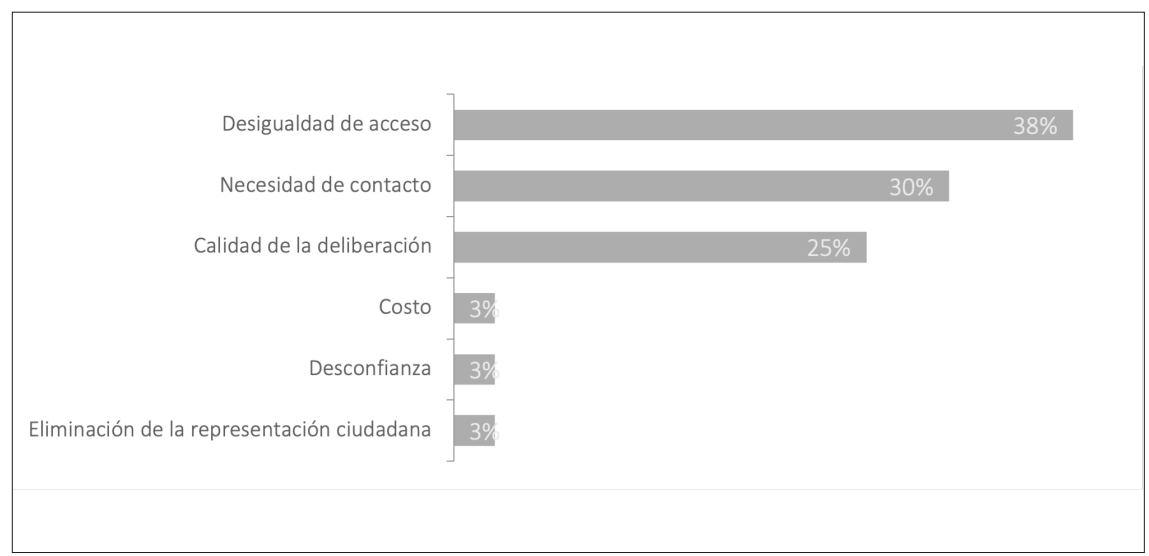

Fuente: elaboración propia sobre la base del relevamiento a municipios.

En la desigualdad de acceso se incluye, nuevamente, el hecho de que existen zonas del territorio con baja conectividad, o que los propios funcionarios municipales no están capacitados, o que para varios grupos de la población no es habitual el entorno digital, o que no todo el mundo dispone de los dispositivos tecnológicos necesarios para poder participar adecuadamente.Veamos algunos de los testimonios en este sentido.

Los funcionarios de Escobar señalaron que con las herramientas digitales: "quedan excluidos quienes presentan dificultades para conectarse a través de plataformas". En General Pico se consideraba que la participación online era: "Problemática en razón a la conectividad de ciertos sectores, limitante en la participación de vecinas y vecinos adultos mayores". El equipo de la Ciudad de Córdoba subrayaba: "La falta o dificultad de acceso a las TICs, a la conectividad y al saber usarlas (generalmente quienes tienen dificultades de acceso son los sectores que ya están excluidos de otros ámbitos de participación, deliberación y decisión) por lo cual se acentúan las diferencias". En la Ciudad de Santa Fe también se enfatizó en el aspecto del conocimiento necesario para la participación online: "Para que sea efectiva necesita cierto entrenamiento en TICs por parte de organizadores y participantes". En la 
misma línea se expresaba desde Concepción del Uruguay: "Excluye este sistema a personas con bajo nivel de educación y a mayores que no están familiarizados con las operaciones online". En la ciudad de Reconquista se señalaba este aspecto de conocimiento, pero también las desigualdades materiales que muchas veces afectan a la población: "La falta de recursos económicos (no disponer de un teléfono o computadora) o la falta de formación en lo digital para poder acceder e interactuar en las plataformas".

En definitiva, la brecha digital tiene tres dimensiones de acuerdo a los testimonios de los equipos municipales: 1) una dimensión socio-económica material (que afecta a los sectores más vulnerables de cualquier franja etaria, en cuanto al acceso a dispositivos y a conexión personal a internet; 2) una dimensión cognitiva, de capacidad, conocimiento o formación (que afecta especialmente a los adultos mayores que no están habituados al uso de las tecnologías); y 3) una dimensión territorial (que afecta a toda la población de ciertas áreas de los municipios en los que no hay buena conectividad).

En segundo lugar, entre las desventajas de la participación online, apareció el hecho de que el contacto con otros en las prácticas participativas resulta ineludible. La ausencia del vínculo personal y del sentimiento de pertenencia es subrayada como un inconveniente de las plataformas digitales, del mismo modo que lo es la falta de contacto directo con el territorio. Como notaban en San Salvador de Jujuy en los formatos online “el vínculo es más distante". Es decir, la participación online construye un tipo de lazo pero uno lejano: no logra producir la cercanía o proximidad que permitiría, a su vez, conocer mejor el territorio, generar compromiso, sentimientos de pertenencia, entusiasmo, y una mejor deliberación.

Estos elementos aparecieron en diferentes testimonios de los equipos municipales. El equipo de Vicente López subrayaba que el contacto presencial redunda en un mejor conocimiento del territorio "hay que conservar instancias presenciales (no obligatorias) para no perder contacto con las distintas realidades del territorio y de las personas que lo habitan. Por ahora, la virtualidad no puede lograr ese contacto". Los equipos de Zárate, La Matanza y Reconquista apuntaban a la incapacidad de generar un sentimiento de pertenencia y de comunidad de los formatos online. Sostenían, respectivamente: "[el formato online] no permite generar el sentimiento de pertenencia y apropiación del proceso..."; "La relación directa y el contacto social son fundamentales para la vida comunitaria"; "[En el formato online] se pierde la interacción cara a cara con el vecino (y todo lo que ello conlleva) y, por otro lado, el hábito de la participación y el compromiso ciudadano". Por su parte, funcionarios de Paraná mencionaban el tema de la movilización de las pasiones, el entusiasmo, que permite la participación "cara a cara" y no es capaz de generar la participación por medio de plataformas digitales: "Se ve fuertemente marcada en la falta de interacción entre vecinos y en el entusiasmo que genera un sistema de votación tradicional". La interacción presencial entre los vecinos redunda, además, en una deliberación de mayor calidad. El equipo de Escobar notaba que "los encuentros 
presenciales facilitan el intercambio mano a mano entre vecinos, sobre todo en la instancia de presentación de propuestas". El equipo de Lanús observaba: "No hay el contacto directo que permite el ida y vuelta de ideas y discusión con los vecinos y el Municipio”. Esto último nos conecta con otra de las desventajas que identificaron los equipos municipales para la realización de PP online: la deliberación.

El empeoramiento de la calidad de la deliberación, en efecto, es la tercera desventaja mencionada. Los equipos municipales consideraron que es mucho más difícil construir ideas colectivas, así como intercambiar experiencias, de manera online. Las plataformas digitales pueden restringir el diálogo y la información compartida debido a los límites en la extensión permitida para publicar contenidos; además, en las reuniones sincrónicas se producen menos intercambios que en los encuentros cara a cara.También observaron que, si bien puede haber numerosos votos, la generación y presentación de proyectos resulta más complicada. El anonimato de la participación online es asimismo considerado como un obstáculo para la buena deliberación. En Gobernador Virasoro, por ejemplo, notaron: "no hay un intercambio más fluido de proyectos y opiniones entre Estado y vecinos". La falta de fluidez de las plataformas digitales se encuentra muy influida, seguramente, por los aspectos asincrónicos de la deliberación, así como por las fallas en la conectividad de las plataformas sincrónicas, que pueden generar delay en el sonido, audios entrecortados, necesidad de desactivar el video, etc.

El riesgo es que un uso no reflexivo de las herramientas digitales sea limitante de la deliberación, como se señalaba en la ciudad de Córdoba: "Las herramientas, si no son bien usadas pueden restringir el diálogo y debate (deliberación)". Los funcionarios de Zárate agregaban: "Dificulta la construcción de ideas grupales y colectivas, y puede suponer una merma en la información a compartir/discutir ya que el formato virtual condiciona la extensión de la información a compartir y discutir". En Tres de Febrero se subrayaba el aspecto informativo de la calidad de la deliberación: "[Las personas que participan] tienen menos conocimiento las propuestas y trabajos de los foros". El equipo de Godoy Cruz señalaba otro aspecto interesante: en los formatos virtuales la atención disminuye rápidamente, de modo que hay que tender a la brevedad de las exposiciones y argumentos: "La virtualidad favorece el intercambio pero no estimula el diálogo. Es importante en lo virtual lo concreto y conciso, por lo que los largos desarrollos de ideas y propuestas pierden rápidamente interés y no pueden plasmar la complejidad de algunas propuestas o proyectos". El anonimato que puede ser inducido por las herramientas digitales era mencionado como otro de los aspectos del empeoramiento de la deliberación, en la medida en que las intervenciones sin rostro y sin el compromiso de "poner la cara" o "poner el cuerpo" parecen tener menos impacto en los demás. En este sentido, afirmada el equipo deVilla Carlos Paz: "La participación por medios digitales es más anónima, por ende, todo aquello que se comparte y se comenta es bajo ese criterio".

Mencionemos, por último, algunas desventajas que fueron nombradas por una minoría de municipios. Primero, llama la atención que el costo o la inversión 
de recursos no haya sido una de las desventajas principales. La pandemia puede haber influido porque fue de la mano con el despliegue de varias herramientas gratuitas. Mientras que anteriormente los gobiernos locales tendían a desarrollar plataformas específicas para la participación online, en 2020 aparecieron opciones que previamente no eran consideradas. Por otro lado, llama también la atención que sea poco apreciada como desventaja la desconfianza en cuanto a la seguridad de los sitios, la validación de los usuarios o la información personal compartida. Por último, lo que algún equipo municipal juzgó como una ventaja, la eliminación de la representación ciudadana, también apareció como una desventaja en el juicio de otro equipo municipal, en la medida en que la participación online impide la mediación de los dirigentes de organizaciones sociales y vecinales que tienen un mayor conocimiento de las necesidades del territorio. Esto fue subrayado por el equipo de General Madariaga, que consideró que una desventaja: "No escuchar y canalizar el reclamo y sugerencias de los presidentes de las Sociedades de Fomento barriales, quienes conocen la realidad de cada barrio y trasladan las inquietudes de sus vecinos".

\section{Conclusiones}

Los resultados de este relevamiento muestran que la pandemia de COVID-19 tuvo un importante impacto negativo en los PP argentinos. Si bien el giro digital se presentó como una alternativa para preservar la participación en un contexto crítico, el proceso de transición de lo presencial hacia lo online no ha sido sencillo y se ha producido en menos de un tercio de los casos. Una opinión compartida, por quienes exploraron el uso de plataformas digitales como por quienes no lo hicieron, es que los formatos online no son suficientes por sí mismos para garantizar fuertes procesos participativos. Aunque los equipos municipales encontraron ventajas significativas en las plataformas digitales (sobre todo que facilitan y permiten sortear obstáculos, que han ofrecido un medio de resistir en la pandemia, o que pueden atraer una participación más masiva), también han identificado desventajas muy relevantes, principalmente las desigualdades de acceso, la falta de contacto personal y territorial y la disminución de la calidad deliberativa.

Las diferencias territoriales que existen en el país pueden influir en las visiones sobre la participación online y sus desventajas. El carácter imprescindible del contacto presencial es subrayado especialmente por los municipios de la provincia de Buenos Aires, mientras que la falta de acceso a las tecnologías o conectividad es señalada sobre todo por municipios de otras provincias, que tienen mayores desigualdades geográficas en cuanto a la infraestructura. Si bien en este trabajo no hemos abordado la cuestión, queda abierto para futuras investigaciones el interrogante sobre el peso de las diferencias geográficas en las experiencias de participación online.

Este relevamiento supone un aporte adicional a las teorías de la deliberación, en la medida en que de los testimonios surge una relación estrecha entre el contacto 
y la calidad de los intercambios. De la comparación entre formatos online y presenciales se desprende que la dimensión afectiva del contacto es un potenciador de la deliberación. Es interesante observar que esta comparación puede ser hecha comprendiendo tanto los formatos online asincrónicos como sincrónicos, porque la pandemia multiplicó la utilización de plataformas que permiten compartir el momento de la enunciación y no mantener conversaciones en diferido pero que, no obstante, siguen careciendo de la dimensión afectiva del contacto presencial. Este aspecto también señala la vía de una profundización de los estudios sobre deliberación, y sobre deliberación online en particular, comparando los formatos tradicionalmente estudiados (por escrito y asincrónicos) con los emergentes a raíz de la pandemia (audiovisuales y sincrónicos).

Otra de las aperturas de este trabajo que contribuye a los estudios sobre deliberación online surge de un testimonio minoritario en el relevamiento, pero muy significativo: el vínculo entre participación digital y eliminación de la representación, con los rasgos positivos y negativos que esto pueda implicar de acuerdo a la concepción de la participación que se sostenga. En definitiva, la participación online tiende a fortalecer la imagen según la cual cada persona participa por sí misma, es decir, una lógica más individual que colectiva. Esto puede ser, al mismo tiempo, más "igualador" y menos "representativo".

En todo caso, este trabajo conduce también a extraer algunas conclusiones prácticas en cuanto a la implementación de los PP en contextos críticos como el de la pandemia: 1) Es conveniente sostener alguna forma de vínculo con la ciudadanía (el que sea posible) para poder recuperar las herramientas participativas plenamente cuando la situación crítica haya pasado;2) Parece favorable incorporar a la propia pandemia -a las preocupaciones, necesidades y temores nuevos que esta genera- entre los temas a discutir, incluso con el propósito de producir un diagnóstico participativo para la gestión local; 3) Lo ideal es tender hacia formas híbridas de participación presencial y digital, combinado etapas de uno u otro formato, incluyendo una instancia de representación o selección de delegados para la dimensión presencial, o simplemente ofreciendo las posibilidades simultáneas de participar por una u otra vía, en la medida en las formas híbridas permitirían equilibrar las desventajas de cada formato y ganar con las ventajas de cada uno. 


\section{Referencias}

Annunziata, R. (2016). "Dinámicas de deliberación ciudadana on-line y off-line". IBEROAMERICANA.América Latina - España - Portugal.Vol 16, Nº 62, pp. 211-216.

Annunziata, R. (2020). "El impacto de los Presupuestos Participativos online en el tipo de proyectos. Un análisis de la experiencia de BAElige". Revista del CLAD. Reforma y Democracia. $N^{\circ} 77$, pp. 157-186.

Arena,E.y Martínez,C. (2013)."Experiencias y buenas prácticas en presupuesto participativo". CABA: UNGS- SRP-UNICEF.

Bhusal,T. (2020). "Citizen participation in times of crisis: Understanding participatory budget during the COVID-19 pandemic in Nepal". ASEAN Journal of Community Engagement. Vol 4, N², pp. 321-341. DOI: https://doi.org/10.7454/ajce.v4i2.1103

Cho, C. H.; Jérôme, T. y Maurice, J. (2020). “'Whatever it takes': first budgetary responses to the COVID-19 pandemic in France". Journal of Public Budgeting, Accounting E Financial Management.Vol 33, $\mathrm{N}^{\circ}$ 1, pp 12-23.

Coleman, S. y Moss, G. (2012). "Under Construction: The Field of Online Deliberation Research". Journal of Information Technology \& Politics. Vol 9, N 1, pp. 1-15.DOI: 10.1080/19331681.2011.635957

Coleman, S. y Cardoso Sampaio, R. (2017). "Sustaining a democratic innovation: a study of three e-participatory budgets in Belo Horizonte". Information, Communication E Society. Vol 20, N 5, pp. 754-769. DOI: http://dx.doi.org/10.1080/1369118X.2016.1203971

Dias, N.; Enríquez, S. y Simone, J. (2019). The Participatory Budgeting World Atlas. Lisboa: Epopeia and Oficina. Disponible en https://bit.ly/2OB7KJ3

Graham, T. (2010). “The Use of Expressives in Online Political Talk: Impeding or Facilitating the Normative Goals of Deliberation?", en Efthimios Tambouris, Ann Macintosh, y Olivier Glassey (eds.) Electronic Participation, Lecture Notes in Computer Science. Berlin, Heidelberg: Springer, pp. 26-41.

Hartz-Karp, J. y Sullivan, B. (2020). “The Unfulfilled Promise of Online Deliberation”. Journal of Deliberative Democracy.Vol 10, № 1, artículo 16, pp. 1-5. https://delibdemjournal.org/ article/id/462/ (el 13 de abril de 2021).

Hernández Trejo, N. E. (2020). "Los desafios para la participación ciudadana desde el contexto de la emergencia sanitaria”. ESPACIO I+D, INNOVACIÓN MÁS DESARROLLO. Vol 9, N²5, pp. 80-98 DOI: https://doi.org/10.31644/IMASD.25.2020.a05 
Manosevitch, I. (2014). The Design of Online Deliberation: Implications for Practice, Theory and Democratic Citizenship. Journal of Public Deliberation, Vol. 10, N 1, artículo 9, pp. $1-4$.

Pineda Nebot, C. (2018). "El presupuesto participativo de Madrid: luces y sombras". Revista de Gestão e Secretariado. Vol 9, № 1, pp. 265-292. DOI: http://dx.doi.org/10.7769/ gesec.v9i1.687

Spada, P.; Mellon,J.;Peixoto,T., y Sjoberg, F.M. (2016).“Effects of the internet on participation: Study of a public policy referendum in Brazil". Journal of Information Technology \& Politics, Vol 13, Nº 3, pp. 187-207. DOI: 10.1080/19331681.2016.1162250

Strandberg, K. y Grönlund, K. (2018). “Online Deliberation”, en Andre Bächtiger, Jonh Dryzek, Jane Mansbridge, y Mark Warren (eds.). The Oxford Handbook of Deliberative Democracy. Oxford: Oxford University Press.

Touchton, M.; Wampler, B. y Spada, P. (2019). "The digital revolution and governance in Brazil: Evidence from participatory budgeting”. Journal of Information Technology \& Politics, Vol 16, N 2, pp. 154-168. DOI: 10.1080/19331681.2019.1613281

UNGS (2015)."Encuesta Nacional de Municipios Argentinos con Presupuesto Participativo”, inédita.

Wampler, B.; McNulty, S. L. y Touchton, M. (2017). "Participatory Budgeting: Spreading Across the Globe". Miami: University of Miami, Boise State University; Franklin \& Marshall College. https://www.transparency-initiative.org/blog/2626/participatory-budgetingspreading-across-globe- $2 /$.

Welp,Y. (2017). “La participación política en la era digital”. Buen Gobierno, N²2, pp. 93-107.

Enviado: 15 de abril de 2021

Aceptado:25 de junio de 2021 